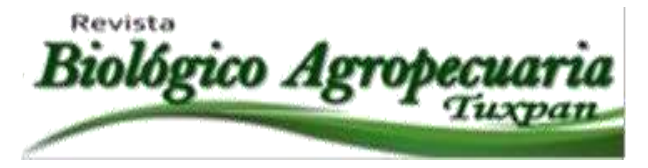

\title{
Estado de la recién creada colección científica de mandíbulas de elasmobranquios (Chordata: Elasmobranchii) de la Facultad de Estudios Superiores Iztacala, UNAM
}

\author{
The status of the jaws collection of Elasmobranchs (Chordata: Elasmobranchii) to the Facultad de \\ Estudios Superiores Iztacala, UNAM \\ Urbán-Minutti Gerardo ${ }^{\bowtie}$ y Luis Fernando Del Moral-Flores \\ Laboratorio de Zoología, Facultad de Estudios Superiores Iztacala, Universidad Nacional Autónoma de \\ México (UNAM), Av. De los Barrios No. 1. Los Reyes Iztacala, 54090 Tlalnepantla, Estado de \\ México, México. \\ ${ }^{\circledR}$ Autor para correspondencia: urbanminutti@hotmail.com
}

Recibido: $07 / 01 / 2016 \quad$ Aceptado: $16 / 06 / 2016$

\section{RESUMEN}

Las mandíbulas son una herramienta importante para el estudio de los elasmobranquios, tanto fósiles como actuales, las cuales difieren entre especies por su tamaño, la forma y número de dientes. Dada su relevancia, en la presente contribución se hace una reseña sobre la creación de la Colección de mandíbulas de Elasmobranquios adjunta a la Colección Ictiológica de la Facultad de Estudios Superiores Iztacala, UNAM. El estado actual del acervo está conformado por 181 mandíbulas, pertenecientes a 28 especies, 18 géneros, 14 familias y 8 órdenes. La familia Carcharhinidae es la mejor representada en cuanto a la riqueza específica ( $7 \mathrm{spp}$.), mientras que el mayor número de mandíbulas pertenecen a las especies Carcharhinus falciformis y Rhizoprionodon longurio, con 85 y 61 respectivamente.

Palabras clave: Colección científica, Condríctios, Mandíbulas, Tiburones, Rayas.

\begin{abstract}
Jaws are an important tool for the study of elasmobranchs, either fossils or existing organisms, all of which differ among species by aspects such as size, shape and number of teeth. Given the relevance, the present contribution contains a review about the formation of the jaws collection of elasmobranchs attached to the FESI (UNAM) ichthyological collection. The current repertory comprises 181 jaws, belonging to 28 species, 18 genera, 14 families and 8 orders. The best represented family is Carcharhinidae with a species richness of $7 \mathrm{spp}$., whereas the largest number of jaws belongs to Carcharhinus falciformis and Rhizoprionodon longurio species, possessing 85 and 61 jaws respectively.
\end{abstract}

Keywords: Scientific collection, Chondrichthyans, Jaws, Sharks, Rays. 


\section{INTRODUCCIÓN}

Las colecciones científicas alrededor del mundo albergan más de 300 años de trabajo sistemático (Martínez-Meyer, 2005). Son el acervo más importante para el conocimiento de la biodiversidad, representan un recurso básico y primordial para la investigación biológica (Cristin y Perrilliat, 2011; Luna-Plascencia et al., 2011). Actualmente son el único recinto que permite tener evidencia física de especies extintas y en peligro, en este sentido dado la crisis de biodiversidad que se padece en la actualidad, su importancia es cada vez más relevante (Cotterill, 1997).

México posee una gran diversidad biológica marina, cuenta con más de $11000 \mathrm{~km}$ de costas y un mar territorial cercano a los 231

$813 \mathrm{~km}^{2}$ (Sarukhán et al., 2009). Parte de esta biodiversidad está representada por los Elasmobranquios, cuya diversidad está compuesta por 206 especies de tiburones y rayas, lo que posiciona a México como una región estratégica en la conservación y protección de este grupo de peces (Del MoralFlores et al., 2015). Son un recurso de gran importancia en cuanto a sus aspectos

biológicos, ecológicos, taxonómicos, biogeográficos y de aprovechamiento (Del Moral-Flores y Pérez-Ponce de León, 2013).

Existen diversas técnicas y estructuras anatómicas que permiten estudiar a los elasmobranquios; una de ellas son las mandíbulas y su dentición, que representan un carácter taxonómico importante, y que ha sido empleado para la clasificación e identificación de diferentes especies (Sadowsky, 1970). La mandíbula se encuentra por debajo del cráneo, está constituida por los cartílagos: palatocuadrado (superior), Meckel (inferior) y el arco hioideo (unión de la mandíbula al neurocráneo). Estos cartílagos sufren una calcificación prismática, llamada "tesserae" (Kemp y Westrin, 1979). Los dientes están unidos a la mandíbula por capas de tejido conectivo (Gajic, 2013) y pueden ser reemplazados en cualquier etapa del desarrollo, pueden tener diferente morfología dependiendo de su alimentación y relación evolutiva (Bass $e t$ al., 1973). El número de dientes en la mandíbula se expresa mediante una formula dental (sensu Applegate, 1965).

Con fines de contribuir en el conocimiento y estudio de los elasmobranquios mexicanos, el presente trabajo enmarca la creación de la colección científica de mandíbulas como parte adjunta de la Colección Ictiológica de la Facultad de Estudios Superiores Iztacala (CIF-I), UNAM. El objetivo de la misma, pretende resguardar el mayor número de especies mexicanas y que sirva como referente de su diversidad e

implementación en futuros estudios comparativos (taxonómicos, paleontológicos, anatómicos, biológicos) y aquellos que coadyuven su aprovechamiento racional, de conservación y protección.

\section{MATERIAL Y MÉTODOS}

La obtención de las mandíbulas se llevó a cabo mediante recolectas efectuadas en algunos sitios de pesca de elasmobranquios en México, en su mayoría provenientes de la pesca ribereña (e.g., Puerto Madero, Chiapas; Puerto Ángel, Oaxaca; Antón Lizardo y Puerto de Veracruz, Veracruz), donde es desechada la cabeza, misma que fue recuperada para la extracción de la mandíbula gracias a la autorización de los dueños de las cooperativas. El muestreo se realizó durante el periodo de diciembre del 2014 a abril del 2016. Los organismos fueron identificados a nivel 
específico mediante claves y guías de campo (Compagno, 1984; Castro-Aguirre y Espinosa, 1996) y en algunos casos, se obtuvieron los datos de longitud total y el sexo.

Se extrajo la mandíbula in situ por medios mecánicos usando cuchillos para separar lo más posible el tejido conectivo y musculo adherido a ella, separando el arco hioidal del cartílago de Meckel y el palatocuadrado. La limpieza final se realizó sumergiendo las mandíbulas en hipoclorito de sodio al $10 \%$, el cual degrada los tejidos, con tiempos de 1 a 5 minutos dependiendo el tamaño de la mandíbula, posteriormente se neutraliza el hipoclorito de sodio con un enjuague de agua corriente de 2 a 5 minutos. El blanqueamiento se realizó con un baño de peróxido de hidrógeno comercial $(3 \%)$ por un período de 5 a 15 minutos. Al finalizar este tratamiento se colocaron durante dos días bajo la sombra, con la mandíbula abierta para su secado. La preservación, etiquetado y almacenamiento por especie (Fig. 1) se llevó a cabo en las instalaciones del Laboratorio de
Zoología de la FESI. La terminología utilizada en éste artículo se basó en los criterios de Mendoza-Vargas y Espinosa-Arrubarrena (2016).

\section{RESULTADOS Y DISCUSIÓN}

El estado actual de la colección está representado por 181 mandíbulas, pertenecientes a 14 especies, 8 géneros, 5 familias y 4 órdenes (Cuadro 1; Fig. 2). Esto representa el $6.5 \%$ de las 214 especies registradas para México (Del Moral-Flores et al., 2015). La familia Carcharhinidae presentó la mayor diversidad específica con 7 especies, mientras que a nivel específico el mayor número de mandíbulas pertenecen al tiburón piloto Carcharhinus falciformis (85), esto coincide con la diversidad observada a nivel de familia y también con la abundancia de captura, siendo esta especie una de la más abundantes en las costas de México y de mayor aporte hace a la captura nacional (Castillo et al., 2002; Soriano-Velásquez et al., 2001; CONAPESCAINP, 2004).

Cuadro 1. Lista sistemática de las especies de Elasmobranquios representadas en la colección de mandíbulas de la CIFI.

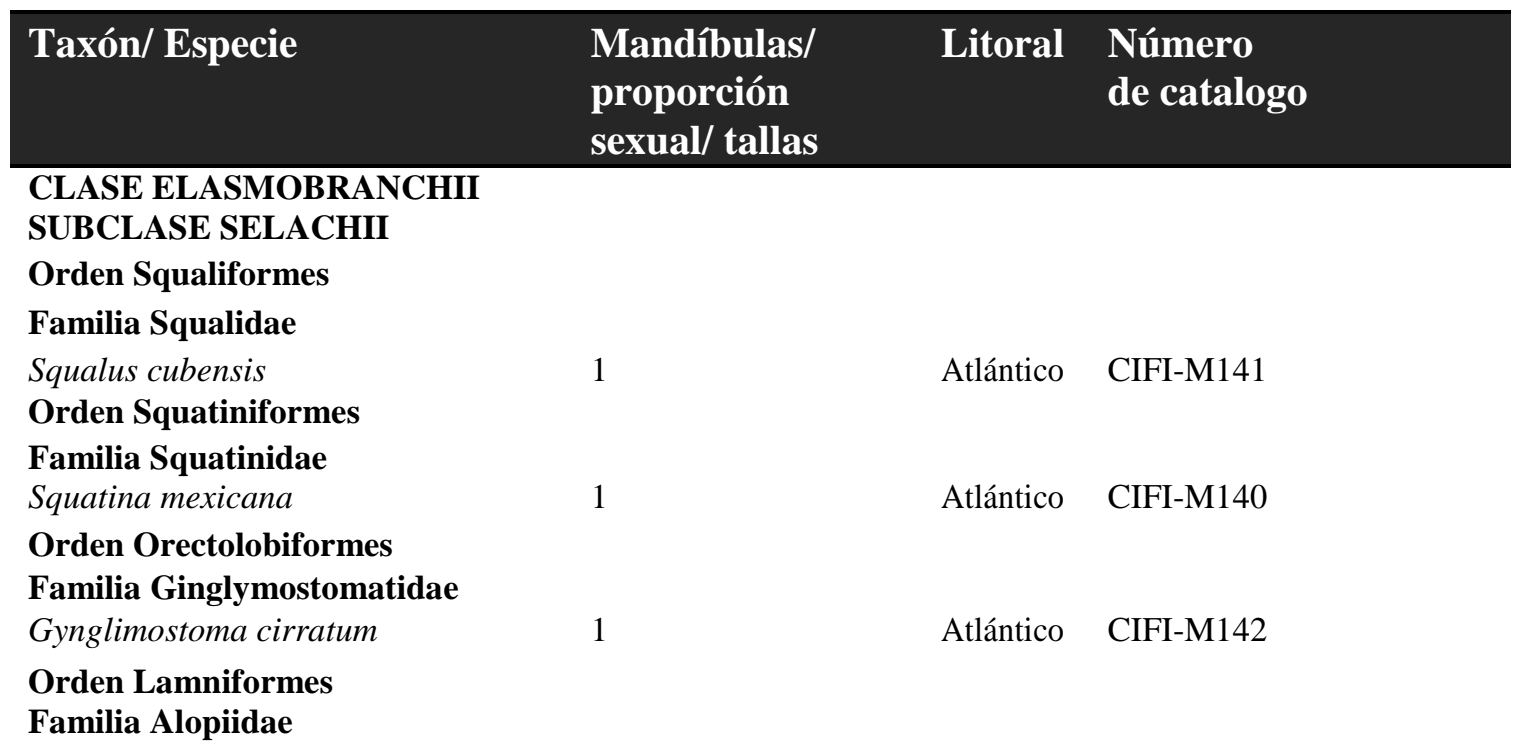




\begin{tabular}{|c|c|c|c|c|}
\hline Alopias pelagicus & 1 & & Pacífico & CIFI-M143 \\
\hline Familia Lamnidae & & & & \\
\hline Isurus oxyrrinchus & 1 & & ¿? & CIFI-M135 \\
\hline Orden Carcharhiniformes & & & & \\
\hline Familia Triakidae & & & & \\
\hline Mustelus lunulatus & 2 & & Pacífico & CIFI-M136, M137 \\
\hline Familia Carcharhinidae & & & & \\
\hline Carcharhinus altimus & 2 & & Atlántico & CIFI-M144, M145 \\
\hline Carcharhinus falciformis & 85 & $\begin{array}{l}42 \mathrm{H}: 43 \mathrm{M} \\
(70-280 \mathrm{~cm})\end{array}$ & Pacífico & $\begin{array}{l}\text { CIFI-M1, M34, M36, M66, } \\
\text { M128, M132 }\end{array}$ \\
\hline Carcharhinus galapagensis & 1 & & Pacífico & CIFI-M133 \\
\hline Carcharhinus limbatus & 5 & & Atlántico & CIFI-M163, M167 \\
\hline Carcharhinus leucas & 3 & & Atlántico & CIFI-M160, M162 \\
\hline Galeocerdo cuvier & 2 & & Atlántico & CIFI-M134, M169 \\
\hline Rhizoprionodon longurio & 61 & $\begin{array}{l}23 \mathrm{H}: 38 \mathrm{M} \\
(37-49 \mathrm{~cm})\end{array}$ & Pacífico & CIFI-M67, M127 \\
\hline Familia Sphyrnidae & & & & \\
\hline Sphyrna lewini & 3 & & Pacífico & CIFI-M35, M138, M139 \\
\hline Sphyrna tiburo & 1 & & Atlántico & \\
\hline SUBCLASE BATOIDEI & & & & \\
\hline Orden Torpediniformes & & & & \\
\hline Familia Narcinidae & & & & \\
\hline Narcine bancrofti & 1 & & Atlántico & CIFI-M147 \\
\hline Narcine entemendor & 1 & & Pacífico & CIFI-M148 \\
\hline Orden Rhinobatiformes & & & & \\
\hline Familia Rhinobatidae & & & & \\
\hline Rhinobatos lentiginosus & 1 & & Atlántico & CIFI-M149 \\
\hline Orden Myliobatiformes & & & & \\
\hline Familia Gymnuridae & & & & \\
\hline Gymnura micrura & 1 & & Atlántico & CIFI-M150 \\
\hline Gymnura marmorata & 1 & & Pacífico & CIFI-M151 \\
\hline Familia Dasyatidae & & & & \\
\hline Dasyatis sabina & 1 & $\begin{array}{l}1 \mathrm{M} \\
(57.5 \mathrm{~cm})\end{array}$ & Atlántico & CIFI-M152 \\
\hline Familia Urotrygonidae & & & & \\
\hline Urobatis jamaicensis & 1 & & Atlántico & CIFI-M153 \\
\hline Urotrygon rogersi & 1 & & Pacífico & CIFI-M154 \\
\hline Urotrygon chilensis & 1 & & Pacífico & CIFI-M155 \\
\hline Familia Rhinopteridae & & & & \\
\hline Rhinoptera bonasus & 1 & & Atlántico & CIFI-M156 \\
\hline Familia Myliobatidae & & & & \\
\hline Aetobatus narinari & 1 & & Atlántico & CIFI-M157 \\
\hline Aetobatus planiceps & 1 & & Pacífico & CIFI-M158 \\
\hline Mobula munkiana & 2 & $\begin{array}{l}1 \mathrm{H}: 1 \mathrm{M} \\
(52-40 \mathrm{~cm})\end{array}$ & & CIFI-M159, M168 \\
\hline TOTAL & 181 & & & \\
\hline
\end{tabular}




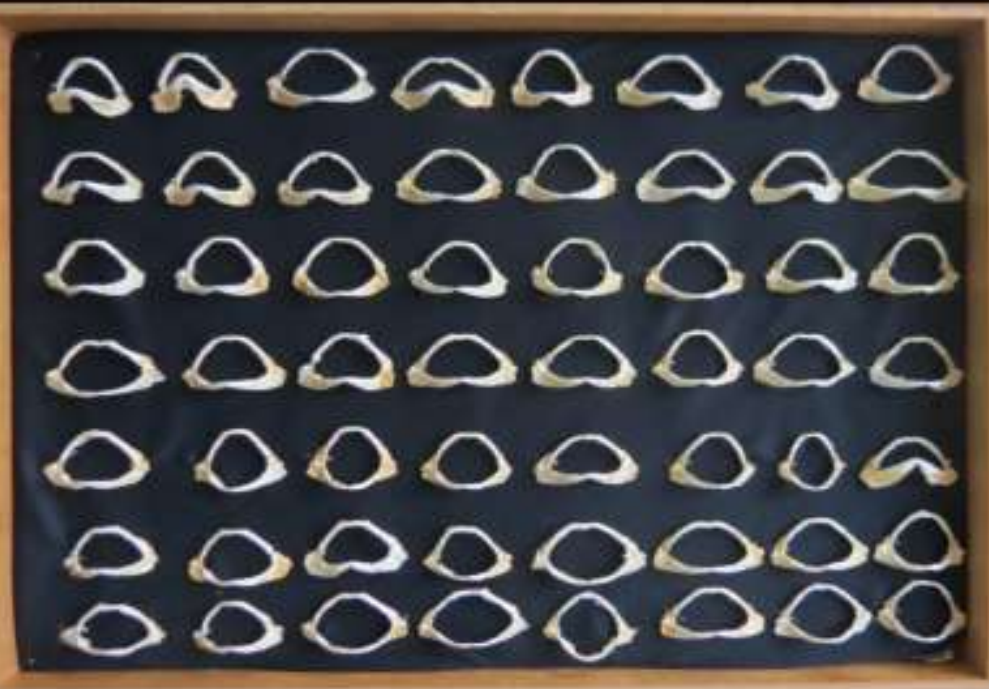

Figura 1. Muestra de mandíbulas del tiburón bironche, Rhizoprionodon longurio, pertenecientes a la Colección de Mandíbulas de la CIFI.

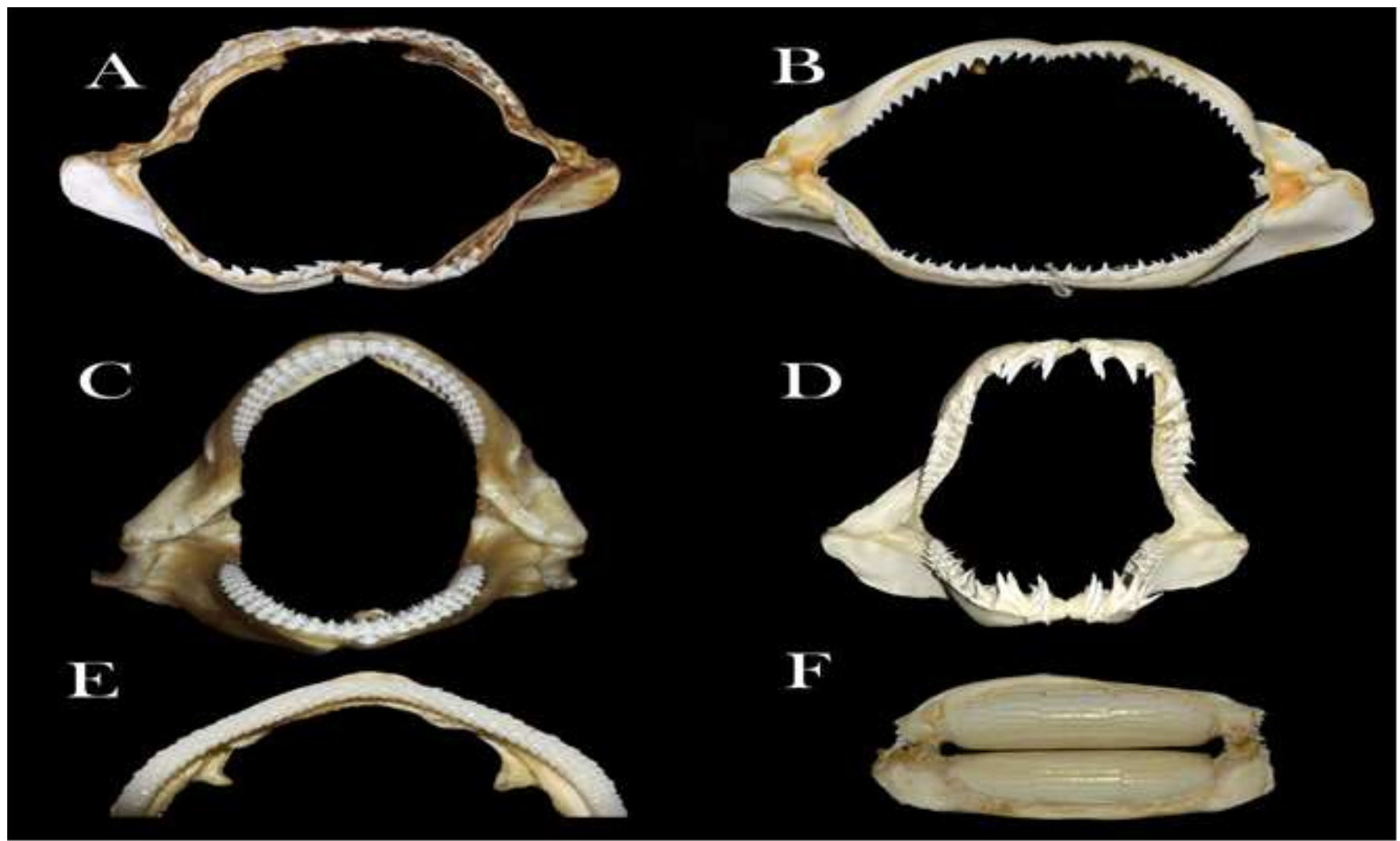

Figura 2. Ejemplo de mandíbulas pertenecientes a diversas especies de Elasmobranquios incluidas en la CIFI. A) Galeocerdo cuvier (Cartílago de Meckel, $\mathrm{CM}=36.6 \mathrm{~cm}$ ); B) Carcharhinus falciformis $(\mathrm{CM}=20.6 \mathrm{~cm}) ; \mathrm{C})$ Gynglimostoma cirratum $(\mathrm{CM}=12 \mathrm{~cm}) ; \mathrm{D})$ Isurus oxyrrinchus $(\mathrm{CM}=18.8 \mathrm{~cm}) ; \mathrm{E})$ Mustelus lunulatus $(\mathrm{CM}=9.4 \mathrm{~cm}) ; \mathrm{F})$ Rhinoptera bonasus $($ Placa dental $=7.8 \mathrm{~cm})$.

Revista Científica Biológico Agropecuaria Tuxpan 4 (1)

ISSN: 2007-6940 
La distinta morfología de los dientes dentro de las especies de elasmobranquios se denomina heterodoncia (Compagno, 1970; Capetta, 1987), con cuatro subdivisiones: la variación puede deberse a diferencias sexuales, heterodoncia sexual (Kajiura y Tricas, 1996), diferentes estadios, heterodoncia ontogénica (Reif, 1984; Applegate y Espinosa-Arrubarrena, 1996), variación en la morfología de los dientes de la mandíbula superior e inferior, heterodoncia dignatica y la variación dental del centro a los extremos de ambas mandíbulas, heterodoncia monognatica (Compagno, 2003). Sin embargo, los datos sobre dichas variaciones dentales son todavía limitados para la mayoría de los elasmobranquios, por lo tanto se realiza una descripción morfológica de los dientes de cada especie ingresada a ésta colección (Cuadro 2) así como una comparativa dental con las demás especies descritas (Fig. 3).

Cuadro 2. Descripción morfológica de los parámetros mandibulares de las especies Carcharhinus

falciformis y Rhizoprionodon longurio, dos especies comunes en las pesquerías mexicanas.

\begin{tabular}{|c|c|c|c|c|c|}
\hline Especie & $\begin{array}{l}\text { Fórmula } \\
\text { dental }\end{array}$ & $\begin{array}{l}\text { Tipo de } \\
\text { heterodoncia }\end{array}$ & $\begin{array}{l}\text { Configuración } \\
\text { de series y filas } \\
\text { dentales }\end{array}$ & Tipo de raíz & $\begin{array}{l}\text { Tipo de } \\
\text { mordida }\end{array}$ \\
\hline $\begin{array}{l}\text { Carcharhinus } \\
\text { falciformis }\end{array}$ & $\frac{16-2-16}{16-1-16}$ & $\begin{array}{l}\text { Dignatica y } \\
\text { monognatica }\end{array}$ & Alterna & Holaulacorriza & $\begin{array}{l}\text { Sujeción y } \\
\text { corte }\end{array}$ \\
\hline $\begin{array}{l}\text { Rhizoprionodon } \\
\text { longurio }\end{array}$ & $\frac{13-2-13}{12-1-12}$ & Monognatica & Alterna & Holaulacorriza & $\begin{array}{l}\text { Corte en } \\
\text { ambas } \\
\text { mandíbulas }\end{array}$ \\
\hline
\end{tabular}
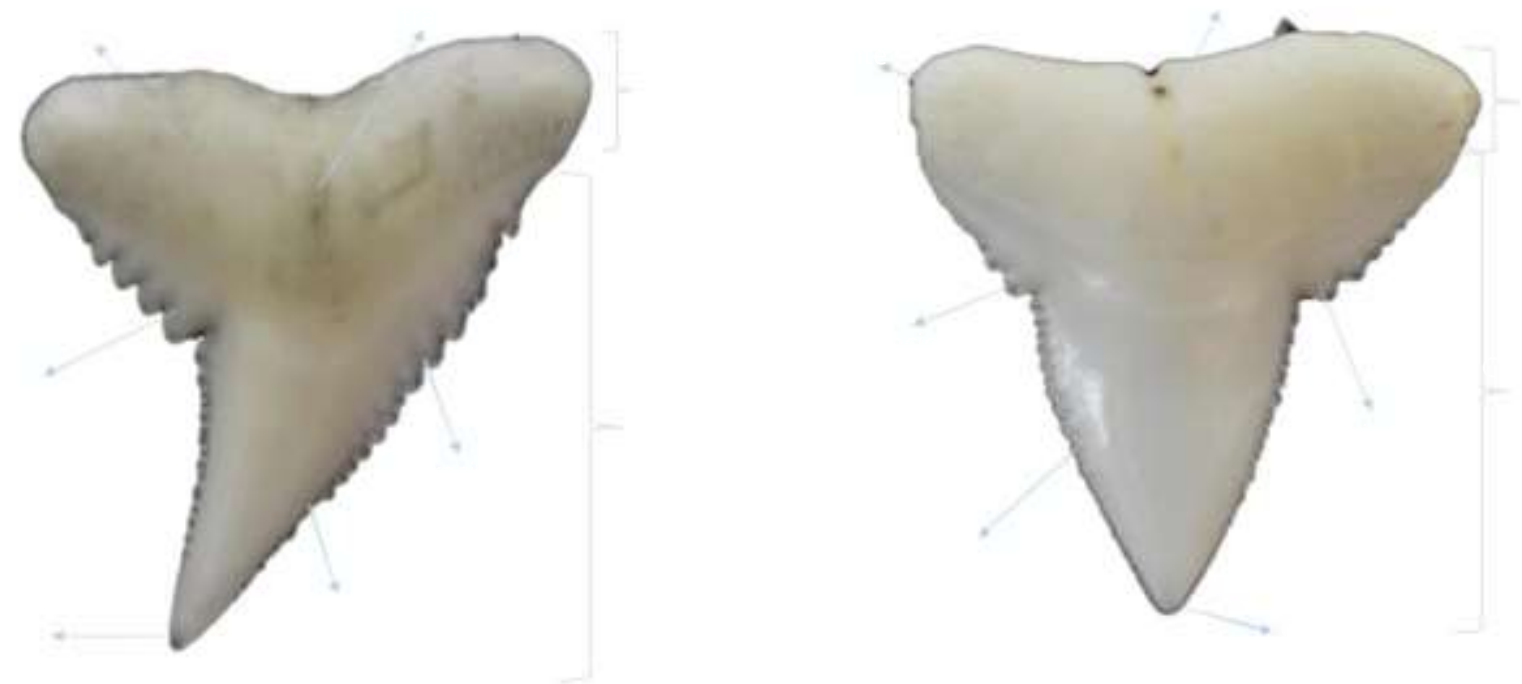

Figura 3. Descripción morfológica dental del tiburón piloto, Carcharhinus falciformis (vista lingual). 
Ésta colección contribuye al conocimiento de los elasmobranquios (Cuadro 2), así como para futuras investigaciones, incluyendo sus implicaciones económicas, educativas, sociales, culturales e históricas (Miller et al., 2004; Suarez y Tsutsui, 2004). Es por ello que se tiene planeado seguir aumentando el acervo, considerando ejemplares de otras latitudes y otras áreas costeras y oceánicas. Otras de las metas de la colección son: apoyar las diversas investigaciones tanto de investigadores reconocidos como de los alumnos adscritos a la FES-I, servir a la conservación y conocimiento de los condríctios mexicanos mediante la divulgación y exposición del material bajo resguardo en diferentes exposiciones, talleres $y$ ferias científicas, creando un vínculo entre la ciencia y la sociedad.

\section{CONCLUSIÓN}

La colección de mandíbulas adjunta de la CIFI, representa una parte importante de la condrictiofauna nacional (12\%) se pretende seguir incluyendo un número mayor de especies para consolidarse como una herramienta importante para el estudio de elasmobranquios en México y como comparación con la información de estos organismos en diferentes partes del mundo.

\section{AGRADECIMIENTOS}

A Andrea Colis por su ayuda y a los revisores anónimos por sus comentarios que enriquecieron esta contribución. Se agradece a los dueños y pescadores de las cooperativas de tiburón. En especial a Manuel Valle y familia, de Puerto Madero, Chis., por la gran ayuda y facilidades durante la estancia en la localidad.

\section{LITERATURA CITADA}

Applegate, S. P. 1965. Tooth terminology and variation in sharks with special reference to the sand shark,

Carcharias taurus Rafinesque. Contributions in Science, Los Angeles County Museum 86: 1-18.

Applegate, S. P and L. Espinosa-Arrubarrena. 1996. The fossil history of Carcharodon and its possible ancestor, Cretolamna: A study in tooth identification. In: A. P. Klimley and D. G. Ainley (EDS.). Great Withe Sharks.

The Biology of Carcharodon carcharias. Academic Press. USA. p. 19 - 36. https://doi.org/10.1016/B978-0

Bass, A. J.; D. D'Aubrey and N. Kistnasamy. 1973. Sharks of the east coast of southern Africa. I. The genus Carcharhinus (Carcharinidae). The Oceanographic Research Institute. Investigational Report 33: 1-168.

Castillo, G. J. L.; V. S. R. Soriano; V. F. Sancho; S. C. E. Ramírez y A. C del Prado-Vera. 2002. Elasmobranquios del Golfo de Tehuantepec, litoral chiapaneco. Universidad Autónoma de Baja California Sur. Informe final SNIB-CONABIO proyecto No. S123: 22-25.

Castro-Aguirre, J. L y H. Espinosa-Pérez. 1996. Listados Faunisticos de México. VII. Catalogo Sistematico de las rayas y especies afines de México. (Chondrychthyes: Elasmobranchii: Rajiformes: Battoideiomorpha). Instituto de Biología. UNAM. p. 75.

Cappetta, H. 1987. Mesozoic and Cenozoic elasmobranchii. Chondrichthyes II. In: H. P., Schultze (ED.). Handbook of paleoichthyology. 3B. Gustav Fischer Verlag, Stuttgart, p. 193.

Compagno, L.J.V. 1970. Systematics of the genus Hemitriakis (Selachii: Carcharhinidae), and related genera. 
Proceedings of the California

Academy of Sciences 38: 63-98.

Compagno, L. J. V. 1984. Sharks of the World.

An annotated and illustrated catalogue

of sharks species know to date.

Carcharhiniformes. FAO Species

Catalogue 4 (Part 2): 251-655.

Compagno, J. L. V. 2003. Sharks of the Order

Carcharhiniformes. The Blackburn

Press. New Jersey, USA. 486 p.

Comisión Nacional de Acuacultura y Pesca e Instituto Nacional de la Pesca (CONAPESCA-INP). 2004. Plan de Acción Nacional para el Manejo y Conservación de Tiburones, Rayas y Especies Afines en México. Comisión Nacional de Acuacultura y Pesca e Instituto Nacional de la Pesca, Secretaría de Agricultura, Ganadería,

Desarrollo Rural, Pesca y Alimentación. Mazatlán, México. 85 p.

Cotterill, F. P. D. 1997. The second Alexandrian tragedy and the fundamental relationship between biological collections and scientific knowledge. In: J.R. Nudds and C. W. Pettitt (EDS). The value and valuations of natural science collections. Proceedings of the International conference, Manchester 1995 The Geological Society, England. 241 p.

Cristin, A. y M. C. Perrilliat. 2011. Las colecciones científicas y la protección del patrimonio paleontológico. Boletín de la Sociedad Geológica Mexicana 63 (3): 4 427. https://doi.org/10.18268/BS

Del Moral-Flores, L. F. y G. Pérez-Ponce de León. 2013. Diversidad de los peces Condrictios (tiburones, rayas y quimeras) de México. Conabio. Biodiversitas 111, 1-6.

Del Moral-Flores, L. F.; J. J. Morrone; J. Alcocer-Durand; H. Espinosa-Pérez y G. Pérez-Ponce de León. 2015. Listado anotado de los tiburones, rayas y quimeras (Chondrichthyes: Elasmobranchii, Holocephali) de México. Arxius de Miscel lània Zoològica 13: 47 - 163.

https://doi.org/10.32800/amz.2015.13.0

Gajic, A. 2013. Comparative odontology of selachians (Chondrichthyes:

Elasmobranchii): developmant and morphological characteristic of teeth. SymbioSE 2013, The 17th anual Symposium Students in Europe, At

The university of Sheffield, Department of molecular biology Sheffiled city, Englad. The Book of abstracts $31-31$.

Kajiura, S. M. and T. C. Tricas. 1996. Seasonal dynamics of dental sexual dimorphism in the Atlantic stingray Dasyatis sabina. The Journal of Experimental Biology 199: 2297 - 2306.

Kemp, N. E. and S. K. Westrin. 1979. Ultrastructure of calcified cartilage in the endoskeletal tesserae of sharks. Journal of Morphology 160(1): 75-101. https://doi.org/10.1002/jmor.1051600106 Luna-Plascencia, R.; A. Castañón-Barrientos y A. Raz-Guzmán. 2011. La biodiversidad en México, su conservación y las colecciones biológicas. Ciencias 101: 36 - 43.

Martínez-Meyer, E. 2005. Las colecciones científicas: eje del conocimiento de la biodiversidad. Revista Mexicana de Mastozoología 9: 4-5.

Mendoza-Vargas, O. U. y L. EspinosaArrubarrena. 2016. Dentición de elasmobranquios, conceptos, patrones, variaciones y terminología. In: L. F. Del Moral-Flores; A. J. RamírezVillalobos; J. A. Martínez-Pérez; A. F. González-Acosta y J. Franco-López (EDS). Colecciones Ictiológicas de Latinoamérica. Facultad de Estudios Superiores Iztacala, Universidad Nacional Autónoma de México y 
Sociedad Ictiológica Mexicana. México. p. 369 - 382.

Miller, B; W. Conway; R. P. Reading;

C.

Wemmer; D. Wildt; D. Kleiman; S. Monfort; A. Rabinowitz; B. Armstrong; and M. Hutchins. 2004. Evaluating the conservation mission of zoos, aquariums, botanical gardens and natural history museums: Conservation Biology 18: 86-93. https://doi.org/10.11 Reif, W. E. 1984. Pattern regulation in shark dentitions. In: G. M. Malacinski and S. V. Bryant (EDS). Pattern formation: a primer in developmental biology. Macmillan Publishing Company, USA. p. $603-621$.

Sadowsky, V. 1970. On the dentition of the sand shark, Odontaspis taurus, from the vicinity of Cananéia, Brazil. Boletim do Instituto Oceanografico, Sao Paulo 18 (10): 37 - 44.

https://doi.org/10.1590/S0373-5524196

Sarukhán, J.; P. Koleff; J. Carabias; J. Soberón; R. Dirzo; J. Llorente-Bousquets; G.
Halffter; R. González; I. March; A. Mohar; S. Anta y J. De la Maza. 2009. Capital natural de México. Síntesis: conocimiento actual, evaluación y perspectivas de sustentabilidad. Comisión Nacional para el Conocimiento y Uso de la Biodiversidad. México. $100 \mathrm{p}$.

Soriano-Velásquez, S. R, A. Solis-Nava, C. Ramirez-Santiago, A. Cid del PradoVera y J.L. Castillo, G. 2001. Tiburones del Golfo de Tehuantepec. In M. A. Cisneros Mata y A. J. Diaz de León (EDS) Sustentabilidas y Pesca Responsable en México 1999-2000. Instituto Nacional de la Pesca. SAGARPA.

Suarez, A.V. and N. D. Tsutsui. 2004. The value of museum collections for research and society: Bioscience. 54: 66-74.

https://doi.org/10.1641/0006-3568(200 


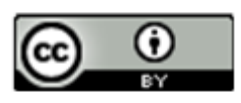

Este tex to está protegido por una licencia licencia CreativeCommons 4.0.

Usted es libre para Compartir —copiar y redistribuir el material en cualquier medio o form ato- y Adaptar el documento —remezclar, transformar y crear a partir del material- para cualquier propósito, , incluso para fines comerciales, siempre que cumpla la condición de:

Atribución: Usted debe dar crédito a la obra original de manera adecuada, proporcionar un enlace a la licencia, e in đicar si se han realizado cam bios. Puede hacerlo en cualquier form a razonable, pero no de form a tal que sugiera que tiene el apoyo del licenciante o 10 recibe por el uso que hace de la obra.

Resumendelicencia - Textocompletodelalicencia 